\title{
Research on the Key Technology of Micro EDM Motion Control System Zhang Liaoyuan $^{1, \mathrm{a}}$, Lv Tingting ${ }^{1, \mathrm{~b}}$, Meng Fanjiang ${ }^{1}$, Ji Zhenming $^{1,}$ Jiang Ruiqi $^{1}$ \\ ${ }^{1}$ Department of Mechanical Manufacturing and Automation, Shenyang Ligong University, Shenyang, 110159, China \\ aemail: zly6217@163.com, bemail: cherish0812@163.com
}

Keywords: motion control system; spark gap; average gap voltage detection

\begin{abstract}
The motion control system of micro EDM CNC milling machine tool which base on the motion control card was designed in this paper. In order to guarantee the processing precision, the discharge gap can be adjust by the analysis and processing of the average gap voltage get through the discharge detection scheme which designed by the analysis of discharge states in EDM.
\end{abstract}

\section{Introduction}

Motion control system is a kind of electric drive control system which refers to the controller as the core, on the basis of automatic control theory and by means of the executing agency such as power electronic devices to control the motor [1]. As the small size of micro EDM (Electrical Discharge Machining) workpiece, the motion control system can be put forward higher requirements to guarantee the processing precision.

The gap effect is that the erosion of the electrode and workpiece will change with the change of discharge gap during the micro EDM process [2]. In order to ensure the machining efficiency and meet the requirement of the machining precision of the workpiece, it is necessary to keep the discharge gap in the appropriate range. Therefore, how to carry on the real-time and accurate measurement of the discharge gap and timely adjustment it through the servo control system has become a top priority of solving the problem of control system.

\section{Design of the Motion Control System}

At present, the open CNC system can be divided into three kinds of structure forms [3]. The first type is a special CNC + PC. Both the PC which is embedded into the special CNC Motion System and the CNC can complete their functions independently at the same time. It has small interference and high reliability, but the new product development cycle is long. The second type is PC+ motion controller. The motion control card which is the core of control is inserted into a slot machine in the general PC which is the hardware platform. Motion control card usually has abundant function library and convenient for the user to carry on the secondary development. The third type is a pure PC. It can complete the processing of all functions of CNC only through the PC. It has strong versatility and flexible programming process. But its development is still not mature enough.

In this paper, the micro EDM CNC milling machine motion control system choose PC+ motion control card and full closed loop motion control method. The diagram of the motion control system shown in Figure 1. 


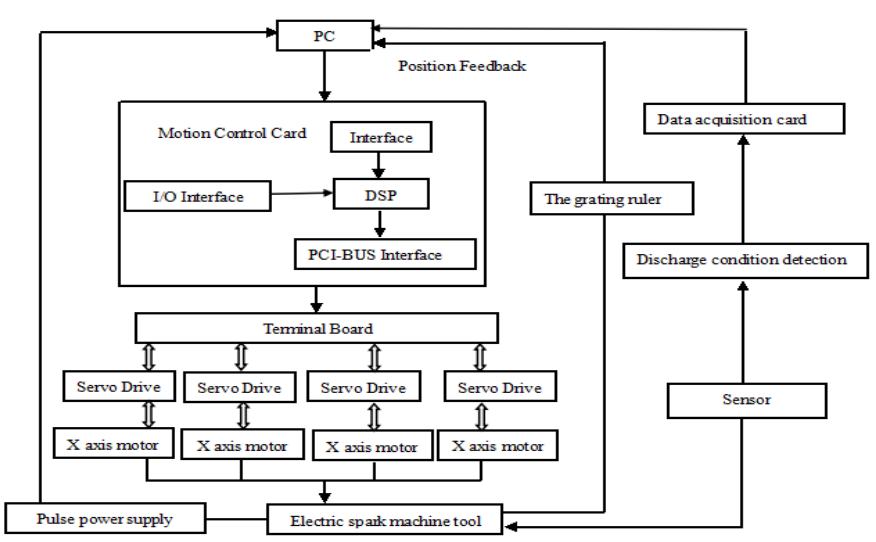

Fig.1. The block diagram of the motion control system

Among them, the PC machine is mainly to complete the document management, parameter setting, system debugging and function. Motion control card interface accepts is sent by the PC include a variety of motion output pulse signal as well as limit switch signal detection, control instruction of [4], and its current state and the related control parameters such as input signals to the PC machine to. The card can realize interpolation and other related logical operations on PC machine, the execution of commands and the I/O management functions. Motion control card can also send control signal to the motor through the terminal board, so the movement of motor can reach the requirement of. Data acquisition card is used to collect sensor to detect the voltage signal gap and give feedback to the PC machine.

\section{Design of discharge gap detection}

EDM is usually divided into open circuit, normal spark discharge, stable arc discharge, transition arc discharge and short circuit five states. Because the micro EDM milling used for the spindle rotation processing method [5], arc discharge basic does not occur, it can be divided into open circuit, short circuit spark discharge and three kinds of state, Its characters are:

(1)Open circuit The gap is not impulse breakdown when the idle state, at this time the big gap between workpiece and electrode wire, good insulation performance, space voltage no-load voltage U0 high resistance, large gap, gap current is zero, does not produce the phenomenon of discharge ablation material. When the servo feed less tracking, namely motor feed speed is less than the cutting speed, there will be no load condition is more, reduce the cutting efficiency.

(2)Normal discharge Across the gap formed in the workpiece and electrode wire with a certain voltage, when the discharge channel is established, the breakdown delay TD, gap breakdown, gap voltage to the discharge sustain voltage UE, gap spark discharge current of IE. This condition often associated with the higher frequency components, the breakdown delay random occurrence, material erosion rate is high, is the ideal state of electric spark line cutting processing.

(3)Short circuit The state space very low resistance, space voltage short circuit voltage us, generally ten volts, when the short-circuit current of is large. There are a small number of high frequency components of the voltage waveform in the gap. The electrical energy consumption under the condition of loop, but the material removal rate is zero, and it will burn the workpiece surface, processing is not stable, increase the probability of broken wire, wire EDM should reduce or avoid the short circuit condition.

Because the actual distance of discharge gap is difficult to measure, and has little significance. But the study found that, the process of EDM discharge gap and the discharge voltage has monotonic relationship between strict. Therefore, we can through the detection of the discharging gap voltage to determine the discharge state and discharge gap, and timely adjust accordingly. This paper uses is the average gap voltage detection method, the system block diagram shown in figure 2. 


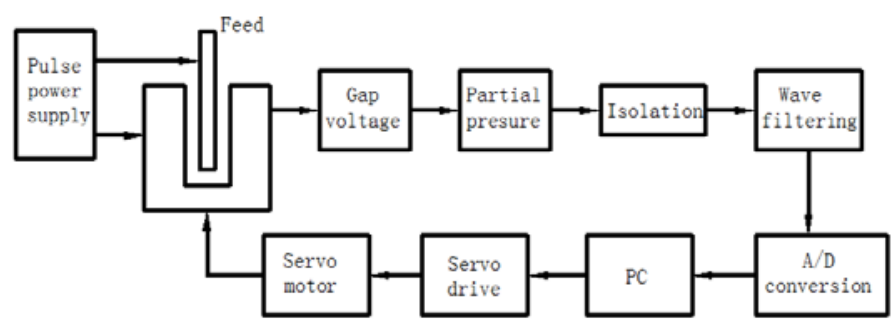

Fig.2. Diagram of discharge condition detection system

With the average gap voltage method for detection of discharge gap, we must first sampled waveform of the discharge gap, the sampling circuit is shown in figure 3:

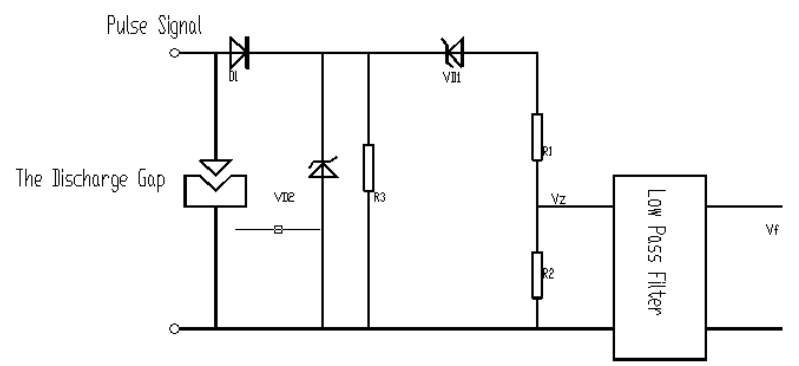

Fig.3. Average gap voltage sampling circuit diagram

Among them, the regulator tube $\mathrm{VD}_{1}$ and $\mathrm{VD}_{2}$ is a voltage controlled in a certain range, and the resistance $R_{1}, R_{2}$ can be divided voltage to the signal acquisition range card can be collected, the high frequency signal through the filter effectively filter circuit in the spark discharge and short circuit state produced, remove the interference signal, make the differential pressure signal becomes smooth, improve the identification accuracy of gap discharge state.

\section{Data processing of discharge gap detection}

In micro EDM process, because of the complexity and instability of the gap voltage signal, deal with the collected processing, in order to ensure its accuracy. In the process of discharge gap voltage instability in [6], the average voltage of the individual from the collected value to determine the discharge condition is easy to cause the error, so using a set of data on methods for the latest acquisition to calculate the weighted average value, the data is new, the higher the weight. The system selected calculation and the average gap voltage of 8 recent data, the formula for:

$$
\bar{X}=\frac{8 x_{7}+7 x_{6}+\ldots+3 x_{2}+2 x_{1}+x_{0}}{8+7+\ldots+3+2+1}
$$

The resulting voltage average value compared with the voltage of $U_{0}$ and $U_{1}$ set, judge its processing discharge state, and drives the motor to make the corresponding adjustment. The servo system control flow chart is shown in figure4:

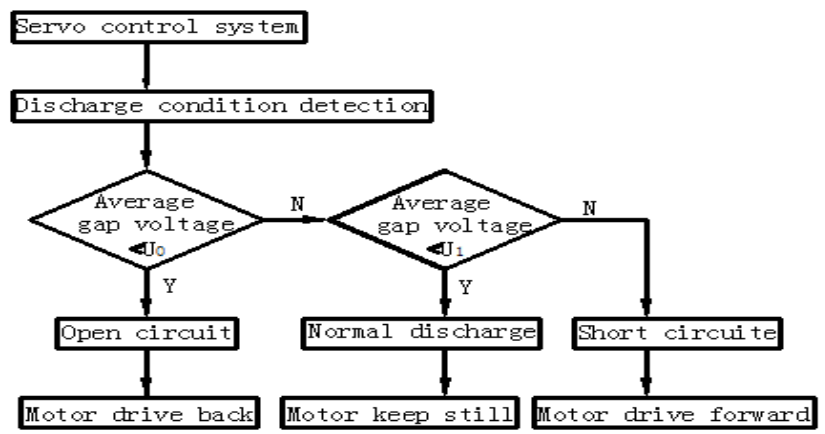

Fig.4. Control principle diagram of servo system 


\section{Conclusion}

The motion control system of micro EDM CNC milling machine tool which base on the motion control card designed in this paper has the advantages of low cost, good performance, easy to develop two times etc.. As the full closed loop control method, the control precision is high. Finished processing design and data on the discharge gap state measuring average gap voltage detection scheme, ensures the detection process stability and accurate, is conducive to the micro EDM CNC milling machine control system stable operation.

\section{References}

[1] Jiajun Wang, Donglian Qi. Development and Prospect of Motion Control System[J]. Electric Age,2004 (10)

[2] W. Meeusen, D. Reynaerts, J. Peirs, H. Van Brussel and etc. The Machining of Free form Micro Moulds by Micro EDM; Work in Progress. Micro mechanics Europe Workshop, Cork, Ireland, 2001, 46 49

[3] Wang J, Ravani B. Computer aided contouring operation for traveling wire electric discharge machine (EDM). Computer Aided Design, 2003, 35 (11): 925-934.

[4] Yong Li, Min Guo, Zhaoying Zhou, Min Hu. Micro electro discharge machine with an inchworm type of micro feed mechanism. Precision Engineering. 2002, 26: 7-1

[5] Yan T, Liao Y S, Chang C C. On-line estimation of work piece height by using networks and hierarchical adaptive control of WEDM. Proceedings of the 13th Electro machining ISEM XIII.Bilbao, SPAIN, 2001, (5): 513-523.

[6] Chaojiang Li, Jicheng Bai, Yongfeng Guo, Zesheng Lu, Jianjun Ding, Monitoring Technology of Gap Discharge Status Based on Floating Threshold for WEDM, 16th International Symposium on Electro machining,2010. 